다(1) $(-) \div$

\title{
TikTok medio de divulgación empleado por los viajeros que realizan turismo sostenible
}

TikTok Means of Dissemination Used by Travelers Who Carry out Sustainable Tourism

Dra. Ariana Paola Herrera Pérez

Profesora titular de la Universidad Metropolitana, Ecuador

www.arianaherrera.com.ec

arherr01@ucm.es

https://orcid.org/0000-0002-5627-1948
Herrera Pérez, A.P., (2021)

Tik Tok medio de divulgación empleado por los viajeros que realizan turismo sostenible

Revista Internacional de Investigación en Comunicación aDResearch ESIC. № $25 \mathrm{Vol} 25$

Monográfico especial, marzo 2021 · Págs. 148 a 159

https://doi.org/10.7263/adresic-025-08 
RESUMEN

\author{
Clasificación JEL: \\ M30, M31, L83 \\ Palabras clave: \\ Redes sociales, \\ experiencia del \\ consumidor, \\ turismo sostenible, \\ TikTok
}

Objetivo: La divulgación del turismo sostenible y el mercado de los viajes se han visto beneficiados por las diversas plataformas sociales. El User-Generated Content se ha convertido en el mecanismo más eficiente para transmitir experiencias de los destinos que visitan. El objetivo de la presente investigación es analizar e identificar la influencia que genera TikTok como medio de difusión del sector.

Diseño/metodología/enfoque: La primera fase metodológica es analítica, que explica el contexto sociocultural y el estado de la cuestión. A través, de los temas más relevantes de turismo sostenible, experiencia de los viajeros, TikTok, vídeos cortos y los hashtags más usados en el sector. La segunda fase metodológica es cualitativa, con enfoque interpretativo; se analizan los vídeos con los siguientes hashtags: \#turismo, \#turismosostenible, \#tiktoktravel, \#travel. En este apartado se aplicará una búsqueda online en formato usuario, incorporando las métricas como número de 'me gusta', comentarios y shares.

Resultados: Los resultados evidencian que TikTok genera un impacto relevante sobre la difusión turística, con énfasis en la promoción del turismo sostenible. Los vídeos con los hashtags más relevantes son los siguientes: \#turismo con 299,8 millones de visualizaciones, \#turismosostenible con un total de 101,6 mil visualizaciones, \#tiktoktravel contiene 20,1 millones de visualizaciones y \#travel con 27,3 millones de visualizaciones. El empleo de esta red social para la publicidad del turismo le da un giro productivo, convirtiéndola en una potente herramienta de divulgación.

Limitaciones/implicaciones: Se considera oportuno realizar el análisis de las métricas de TikTok por país, debido a que la implementación de la plataforma se encuentra desarrollada en distintos niveles por parte de los usuarios y los establecimientos. La red social, por el momento, sólo cuenta con un único mecanismo de análisis de las estadísticas y es la analítica de las cuentas Pro, que al ser de carácter privado de los usuarios no permite realizar un análisis profundo de las métricas.

Originalidad/contribución: La investigación se caracteriza por ser pionera en el análisis del impacto de TikTok en la divulgación del turismo sostenible, mediante la revisión de los vídeos cortos con mayor impacto de visualizaciones del sector.

\section{ABSTRACT}

\section{JEL Classification: M30, M31, L83 Key words:} Social media, consumer experience, sustainable tourism Tik Tok
Purpose: The dissemination of sustainable tourism and the travel market in general has benefited from the various social platforms. The User-Generated Content has become the most efficient mechanism for transmitting experiences of the destinations they visit. Therefore, the objective of this research is focused on analyzing and identifying the influence generated by TikTok as a means of disseminating the sector.

Design / Methodology / Approach: The first methodological phase is analytical, which explains the sociocultural context and the state of the question. Through, the most relevant topics of sustainable tourism, traveler experience, TikTok, short videos and the most used hashtags in the sector. The second methodological phase is qualitative, with an interpretive approach; The videos are analyzed with the following hashtags: \#turismo, \#turismosostenible, \#tiktoktravel, \#travel. In this section, an online search will be applied in user format, incorporating metrics such as number of likes, comments and shares.

Results: The results show that the TikTok platform generates a relevant impact on tourist diffusion with an emphasis on promoting sustainable tourism. The videos with the most relevant hashtags are the following: \#turismo with 299.8 million views, \#turismosostenible with a total of 101.6 thousand views, \#tiktoktravel contains 20.1 million views and \#travel with 27.3 million of visualizations. The use of this social network for tourism advertising gives it a productive turn, turning it into a powerful dissemination tool.

Limitations / Implications: It is considered appropriate to perform the analysis of TikTok metrics by country, because the implementation of the platform is developed at different levels by users and establishments. However, the social network at the moment has a single mechanism for analyzing the statistics and that is the analytics of the Pro accounts, which being private to the users, it is not possible to carry out a deep analysis of the metrics.

Originality/Contribution: The research is characterized by being a pioneer in the analysis of the impact of TikTok in the dissemination of sustainable tourism, by reviewing the short videos with the greatest impact of views related to the sector. 


\section{Introducción}

A raíz de los últimos acontecimientos que la humanidad atraviesa, derivados de la pandemia mundial, el turismo es uno de los sectores de la economía de cada país que se ha visto afectado significativamente durante el 2020 y 2021. Según la Organización Mundial del Turismo, durante el 2020, la reducción de turistas internacionales oscila entre el $60 \%$ al $80 \%$, una pérdida de ingresos entre 910 mil millones y 1,2 billones de dólares y una pérdida de empleos de 100 a 120 millones (OMT, 2020). La reducción de la movilidad humana y el riesgo que implica el desplazamiento masivo de turistas ha contribuido en el fomento de otras formas de hacer turismo. Tienen especial énfasis las opciones que facilitan el esparcimiento en áreas abiertas y el contacto con la naturaleza. Es así que el turismo sostenible cada vez toma más protagonismo a nivel de los destinos, establecimientos y viajeros. El cuidado de la naturaleza y a su vez, el brindar una experiencia holística hacia los consumidores es el enfoque de la propuesta de valor que diversos establecimientos brindan a sus clientes.

La difusión del turismo sostenible y el mercado de los viajes en general se han visto beneficiados de las diversas plataformas sociales. La interacción entre los usuarios se ha convertido en el mecanismo más eficiente para transmitir experiencias en los diferentes destinos y propiedades que visitan. Sin embargo, la disruptiva red social TikTok acapara la atención de los internautas y es una de las aplicaciones móviles con más creciente popularidad y descargas (Shutsko, 2020). Su característica principal yace en vídeos muy cortos y sonidos de tendencia, con los que se pueden compartir vivencias cotidianas o recreadas (Rey, 2020). Además, promueven que otros usuarios realicen réplicas de esos vídeos virales, de forma individual o en dúos.

Los principales factores que influyen en la experiencia del usuario en TikTok son los emocio- nales, usabilidad e interactivos, con la siguiente valoración porcentual: «Los factores emocionales representan el 48,77\%, los factores interactivos el 31,23 \% y los factores de usabilidad el 20,00 \%» (Feng, Chen y Wu, 2019, p. 55). Es así, que se recomienda que las campañas publicitarias de viajes estén acompañadas de una alta dosis de emotividad, para generar un impacto significativo en la audiencia.

Cada vez son más los viajeros que optan por esta red social para transmitir rápidamente aspectos puntuales de sus viajes y estilo de vida. Las fotos eran la mejor forma de compartir lugares de interés. Sin embargo, la opción de vídeos cortos ha generado especial revuelo entre centennials y millennials. Millones de vídeos se suben día a día a la red social y se incrementa el número de perfiles. En la actualidad, ya existen influencers de esta red que generan contenido especializado, cautivando la atención y promoviendo la réplica de los mismos.

Diversos lugares del mundo se han convertido en tendencia gracias a TikTok, el uso de hashtags específicos como \#turismo, \#turismo sostenible, \#travel y los nombres de las ciudades y países, que constituyen el formato más frecuente de referencia en los vídeos cortos. En el sector del turismo, los vídeos que se popularizan son de los viajeros con fondos de los destinos. Por esta razón, los TikTokers buscan espacios o lugares que cuenten con bondades naturales únicas o poco comunes.

Por todo lo anterior, la presente investigación se centra en analizar el impacto de esta plataforma social en la difusión de las experiencias de los viajeros que hacen turismo sostenible.

\section{Marco teórico}

\subsection{Turismo Sostenible}

Con el surgimiento de nuevos sectores en la economía del mundo, el sector turístico se ha conver- 
tido en un medio de generación de recursos para diferentes países. En algunos casos, es la principal fuente de riqueza y todo se presenta en relación de las potencialidades y bondades que poseen los destinos. Sin embargo, no se puede ignorar que esta actividad debe ser administrada y desarrollada de forma sostenible para evitar un potencial desequilibrio social y natural (Linares y Garrido, 2014).

Para generar la sostenibilidad de un destino se debe implementar un enfoque sistémico. Sólo así los recursos existentes pueden lograr un alto nivel de satisfacción en los viajeros. A pesar de los esfuerzos que los establecimientos realizan por implementar procesos alineados con la sostenibilidad, aún resulta compleja la identificación y valoración de este concepto por parte de los usuarios (Cabrer et al., 2010).

Para lograr que el turismo sostenible se posicione en la mente del consumidor y se convierta en un requerimiento en los destinos, es necesario que los establecimientos promuevan actividades de interés de los stakeholders. Es así, como se podrá fortalecer un sistema que genere vínculos intangibles con los grupos de interés (Alzate y Orozco, 2018).

\subsection{Experiencias de los viajeros}

Las necesidades de los viajeros cada vez se vuelven más aspiracionales. La búsqueda de atención, reconocimiento y vivencias inolvidables son los factores más influyentes a la hora de escoger un destino (Herrera, 2020). Ante este requerimiento, nacen las experiencias holísticas, como el medio que permite involucrar los cinco sentidos y hacer que la vivencia se vuelva digna de ser recordada. Para ello, es importante buscar tematizar la experiencia y armonizarla con aspectos positivos, involucrar los sentidos y eliminar todo cuanto pueda obstruir el proceso de disfrute del turista (Pine y Gilmore, 1998).
El diseño de la experiencia del consumidor es clave en la definición de la propuesta de valor que se oferta a los viajeros. Es el mecanismo que va a delimitar los aspectos que fomentan interés en los consumidores (Liu et al., 2020). Tampoco se puede olvidar el alto compromiso en el cumplimiento de la oferta realizada, para difundir una promesa consistente durante de la difusión online y la vivencia del servicio en el punto de consumo. Aquí toma especial interés la investigación previa que los viajeros realizan en las diferentes plataformas sociales.

Por estas razones se debe tener especial cautela en el tipo de contenido que se comparte en las redes sociales. Hay que evitar caer en el sobredimensionamiento de las localidades o añadir complementos efectistas y poco realistas sólo para la producción audiovisual. Es necesario ser objetivos e imparciales para evitar causar un impacto negativo en los viajeros (Rialti et al., 2018). Es necesario recordar que uno de los principales intereses de los turistas durante los viajes es generar contenido fotográfico y de vídeo para compartir en sus cuentas personales. Compartir experiencias en diversos formatos es un factor común en este sector y con el surgimiento de la plataforma TikTok se ha incrementado sustancialmente.

\subsection{TikTok una oportunidad para el turismo}

Las personas emplean cada vez más tiempo buscando información en línea. El 59 \% de la población mundial está activa en alguna plataforma social (Dwivedi et al., 2020). Con el confinamiento la estadística de la red social TikTok sigue en crecimiento y en especial, la generación de contenido de viajes se difundió con vídeos cortos desde el lugar en que se encontraban los usuarios. Una de sus desventajas más comentadas es la deficiencia para controlar y prohibir vídeos groseros o vulga- 


\section{Imagen 1. Formato de la presentación de las métricas en Tik Tok}

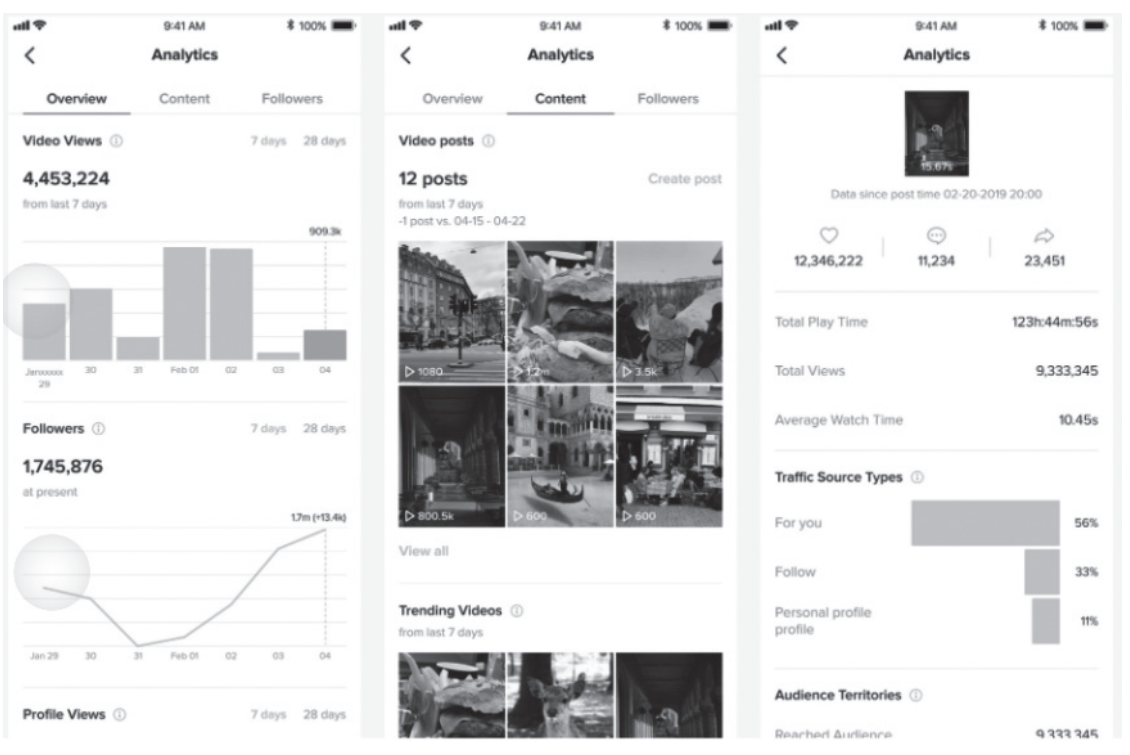

Fuente: Página oficial de Tik Tok (2020). Recuperado de: https://newsroom.tiktok.com/en-us/pro-accounts-offer-new-insightsto-avid-creators/.

res, generando incomodidad en muchos usuarios (Dai y Chen, 2019). No obstante, sus ventajas poseen mayor valor y destaca la versatilidad que provee la plataforma para la creación de vídeos cortos de 30 y 60 segundos, con las opciones de filtros y edición totalmente gratuitos.

Además, facilita que los usuarios se sumerjan en el área multimedia, tomando especial interés entre las generaciones $Z$ y millennial. Sin embargo, a los gerentes de marketing de sectores como el de alimentos y bebidas, viajes, entretenimiento y afines, les cuesta tomar decisiones rápidas y acoplarse a la veloz evolución que los usuarios de este entorno viven (Haenlein et al., 2020).

Esta plataforma social se ha convertido en un potente medio de difusión para el sector turístico. Según datos de la empresa estadounidense de investigación de mercados Forrester Research (2020), las publicaciones en TikTok permite 58 veces más de interacciones que Facebook y 150 veces más que Twitter. A raíz de la escalada exponencial de impacto en los usuarios, empresas de todo el mundo inauguraron sus cuentas e incluso, algunas consideraron esta red social en sus planes de marketing para el 2021.

Es relevante destacar que las estrategias de marketing deben ser específicas para el segmento de usuarios que interactúan en la misma, quienes en su mayoría se encuentran entre las edades de 16 a 25 años. Asimismo, se concentran en las intenciones de viaje de los jóvenes turistas, quienes poseen tres principales motivaciones: ampliación del conocimiento, relajación y autorrealización (Ying y Krishnapillai, 2018). 
Con la escalada de la plataforma, surgió la necesidad de análisis de la evolución de la misma en las cuentas privadas. Por esta razón, desde la página oficial de TikTok (2019), se indica que los usuarios solicitaron una opción que les permita identificar información de su audiencia, rendimiento e impacto de las publicaciones. En base a este pedido, implementaron la opción de Pro Account, una herramienta que facilita obtener métricas de tres aspectos:

- Resumen: Este apartado muestra estadísticas de seguidores, visualizaciones de vídeo y de perfil en dos opciones: 7 días o 28 días.

- Contenidos: Publicaciones de vídeo, vídeos con sus respectivas estadísticas de número de likes, comentarios, shares, reproducciones, visualizaciones, georreferenciación de la audiencia y vídeos que se encuentran en tendencia.

- Seguidores: Esta sección contiene datos relevantes de la audiencia como sexo, ubicación, actividades que realizan o sonidos más escuchados.

Los establecimientos turísticos que disponen de una cuenta Pro pueden acceder a todas las métricas mencionadas. Datos que influyen de forma positiva en la planeación estratégica y operativa del contenido.

La metodología de promoción de las propiedades ha evolucionado y quienes aún se mantienen con la forma tradicional de difusión, ya no es atractiva para los viajeros; quienes buscan vivir una experiencia diferenciadora, sentirse especiales y atesorar recuerdos para compartir. La opción de los vídeos cortos y los beneficios de edición que contiene TikTok ha conquistado a los internautas. Por ende, para ellos es la mejor forma de compartir sus vivencias, autoexpresarse, interactuar con la sociedad y escapar de la presión del día a día (Omar y Dequan, 2020).

\subsection{Los hashtags más empleados en el turismo}

En junio del 2019, TikTok lanzó la campaña \#TikTokTravel como un reto a nivel mundial. La misma fue desarrollada en 100 países con el objetivo de compartir la diversidad que tiene el planeta. El reto planteado por la plataforma debía incluir la canción Summer, con la finalidad de que existiera un elemento en común entre los millones de contenidos que se subieron por parte de los TikTokers. Algunos países adoptaron sus propios hashtags como \#ThisisJapan, \#ThisisMadrid, \#ExperiencePenang. Es importante destacar que al cierre del 2019, el hashtag \#TikTokTravel llegó a 6,2 millones de visitas (Hotelmize, 2019).

En Latinoamérica el primer hotel resort en abrir su cuenta fue @FairmontMayakoba. Su objetivo era atraer a la mayor cantidad posible de turistas a la Riviera Maya. Promueve la interacción con su audiencia generando desafíos que contemplan condiciones específicas. Además, premian a los creadores de contenido relevante con estadías en su establecimiento. Como resultado de estas propuestas han conseguido un alto nivel de interacción con su audiencia.

Otro subsector que posee notoriedad en la plataforma es el de alimentos y bebidas, en el que los vídeos cortos se difunden masivamente (Li et al., 2018). Además de las acciones promovidas por la red social para promover el turismo, los viajeros han empleado sus propios hashtags, como \#turismo, \#turismosostenible, \#travel y \#experience; añadiendo los hashtags tradicionales con los nombres del destino donde se encuentran.

Los creadores de contenido buscan espacios abiertos y en especial, con riqueza natural que les permita, a través de la biodiversidad, tener el escenario perfecto para la generación de los vídeos. La búsqueda de transmitir experiencias diferenciadoras y que aporten valor ha fomentado 
la difusión del turismo sostenible, el cual se centra en disfrutar de la naturaleza, cuidarla y a su vez, brindar una vivencia holística a los turistas.

\subsection{Vídeos cortos de TikTok y la promoción de los destinos}

Los vídeos cortos llegaron para quedarse por una larga temporada, con énfasis en los usuarios entre 16 a 30 años. Quienes están dispuestos a crear vídeos en mayor proporción son la generación Z y millennials, debido a cuán atractivo les resulta el área multimedia. Sin embargo, todas las edades disfrutan visualizándolos. Gracias a la facilidad de descarga, estos vídeos se comparten rápidamente en diferentes plataformas como WhatsApp, Facebook, Instagram y su sección de stories y estados. La tecnología brinda las facilidades para co-crear experiencias, con especial énfasis en el sector del turismo (Zhang et al., 2018).

TikTok ha representado una gran oportunidad para diversas industrias en especial la del turismo, como generador de propaganda de la marca ciudad (Zhang, 2020). Los destinos y establecimientos turísticos ven esta plataforma como un medio estratégico que permite fomentar una positiva forma de viajar. Los vídeos cortos permiten compartir las bondades del destino y transmitir emociones, incluso generar sensaciones en quien los visualiza. Aquí toman relevancia las experiencias holísticas que genera el turismo sostenible, creando un alto grado de fidelización y conexión con los viajeros, en especial cuando las vivencias son memorables.

El nivel de impacto que un vídeo alcanza sobre un viajero es superior que el de las fotografías en sus diferentes formatos. Por ende, el recuerdo del destino, recompra de servicios y recomendación de los diferentes establecimientos son superiores. Las empresas que se encuentran en constante innovación apuestan por los nuevos formatos de difusión y las diferentes metodologías disruptivas de llegar al consumidor. Un área que impacta positivamente TikTok en el individuo es la comunicación y la colaboración, gracias a que después de producir el vídeo se lo comparte con millones de usuarios del área de interés (Carrión, 2020).

Es importante buscar la adaptación para la supervivencia en el mercado turístico. Los jóvenes e intrépidos viajeros van a apostar constantemente por las nuevas tendencias de viajes y de comunicación entre sus comunidades digitales. El camino correcto es estar donde los consumidores y clientes potenciales se encuentran. TikTok se ha convertido en una plataforma que fomenta la comercialización de los diferentes formatos de hacer turismo, entre ellos el turismo rural, y trae consigo un incremento de la demanda de esta forma de disfrutar de la naturaleza (Shen, 2019).

\section{Metodología}

La presente investigación se centra en dos fases:

La primera fase metodológica es de carácter analítica, que explica el contexto sociocultural y el estado de la cuestión.

Tiene como finalidad la recopilación de la información más relevante de turismo sostenible, experiencia de los viajeros, TikTok y los hashtags más usados en el sector, a través de su uso por parte de autores contemporáneos que explican el impacto de la red social en la experiencia de los viajeros.

La segunda fase metodológica es cualitativa, porque se analizan los vídeos y hashtags más significativos de las variables de estudio.

En este apartado se aplicará una búsqueda online en formato usuario, de los vídeos y hashtags más significativos de la industria del turismo, incorporando las métricas como número de me gusta, comentarios y share. El objetivo es explicar y comentar la influencia que genera TikTok en el sector del turismo sostenible. 


\section{Análisis y resultados}

\subsection{Vídeos y hashtags de mayor tendencia en TikTok}

En el presente apartado se analizan los vídeos y hashtags de tendencia en el sector del turismo, turismo sostenible y viajes; además de las características de los vídeos cortos que lideran en métricas en TikTok, a partir de temas previamente expuestos.

El primer hashtag identificado es \#turismo. El mismo dispone de 299,8 millones de visualizaciones al 3 de enero del 2021. El vídeo corto más significativo con este hashtag proviene de la cuenta@edwinsevero, que recoge un total de 600,1 mil 'me gusta', 3261 comentarios y 3267 shares. El área donde fue grabado es en el «anillo de la vida» o el «anillo de la armonía», ubicado en China, en la ciudad de Shenfu. Numerosos viajeros comentan que es un camino para llegar a otra dimensión o que permite conectarse con el cielo.

El segundo hashtag estudiado es \#turismosostenible, con un total de 101,6 mil visualizaciones, al 3 de enero de 2021. El vídeo corto más relevante proviene de la cuenta pública de @oscarrasquilla, con un total de 623 «me gusta, 9 comentarios y 23 shares. El lugar donde se realizó la grabación es el volcán Barú, ubicado en Panamá, en la provincia de Chiriquí. El mismo es visitado por turistas de todo el mundo, quienes buscan captar la mejor toma fotográfica desde los increíbles paisajes y vistas que posee, (Véase figura 3, pag. 156).

El tercer hashtag que se analizó es \#travel, una palabra clave empleada de forma significativa a nivel mundial en el sector de los viajes. Posee 27,3 millones de visualizaciones al 3 de enero de 2021. Uno de los vídeos de mayor tendencia proviene de una cuenta especializada en viajes, denominada@movchal_travel, que contiene un

\section{Imagen 2. Collage del hashtag \#turismo en Tik Tok.}
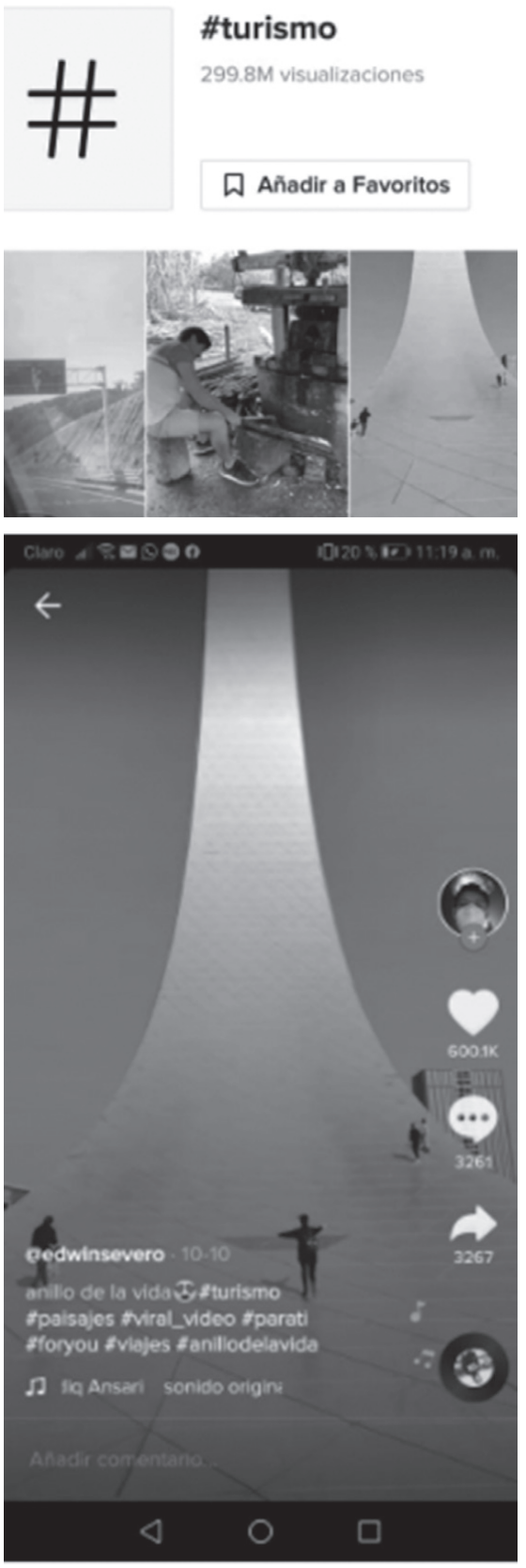

Fuente: Página oficial de TikTok, 3 de enero de 2021. 


\section{Imagen 3. Collage del hashtag \#turismosostenible en Tik Tok}

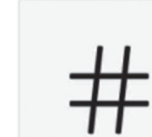

\section{\#turismosostenible}

101.6K visualizaciones

Ф Añadir a Favoritos
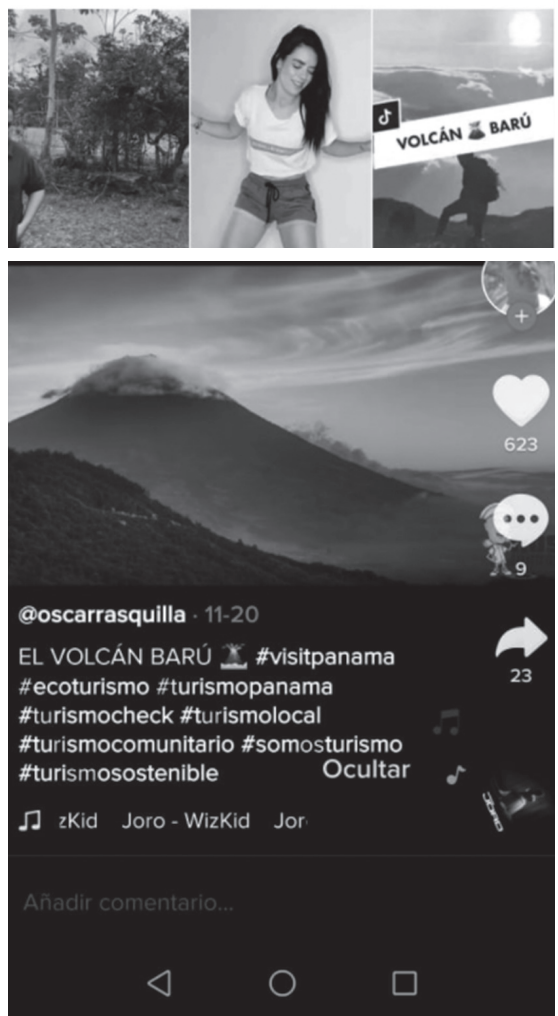

Fuente: Página oficial de Tik Tok, 3 de enero de 2021.

total de 3,8 millones de «me gusta», 18,7 mil comentarios y 103,5 shares. El escenario donde se realizó la grabación fue Suiza, y la viajera promueve su experiencia a bordo de un carrito tipo tren al aire libre, que desciende desde una montaña.

El último hashtag, y no menos importante, es \#tiktoktravel, que nació a raíz del challenge propuesto por la misma plataforma, con el ob-

\section{Imagen 4. Collage del hashtag \#travel en Tik Tok}

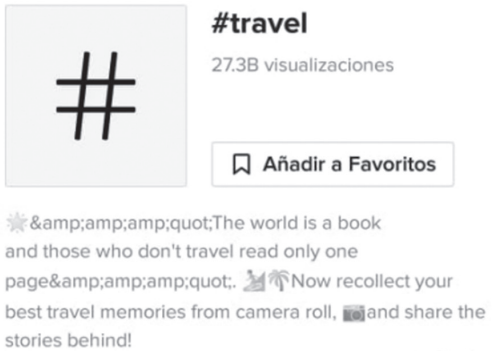
stories behind!

Reducir $\mathbf{A}$
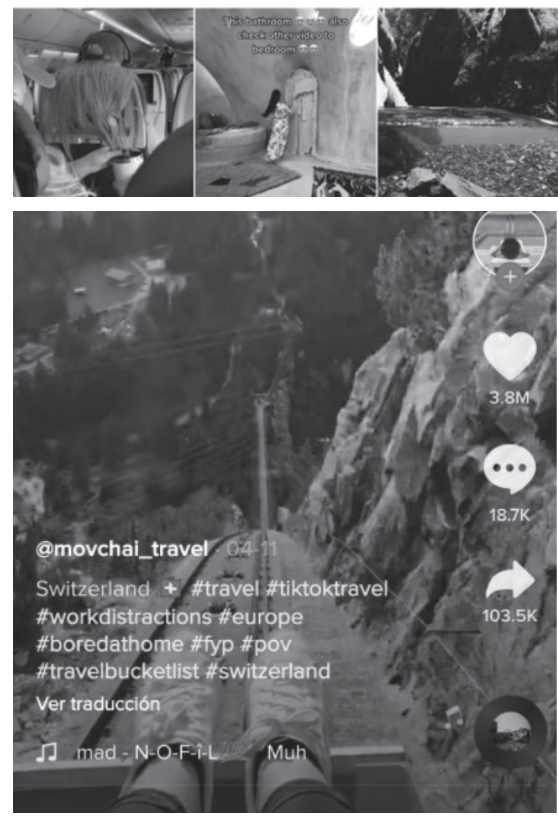

Fuente: Página oficial de Tik Tok, 3 de enero de 2021.

jetivo de motivar la difusión de los destinos por los generadores de contenido. Es así que miles de TikTokers del mundo, desde su creación en el 2019, han generado contenido y lo siguen haciendo nombrando el hashtag de la campaña.

A 3 de enero del 2021, el hashtag \#tiktoktravel contiene 20,1 millones de visualizaciones y uno de los vídeos de mayor tendencia proviene 


\section{Imagen 5. Collage del hashtag \#travel en Tik Tok}

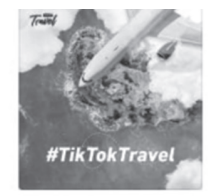

\section{\#tiktoktravel}

$20.1 \mathrm{~B}$ visualizaciones

ヤ Añadir a Favoritos

Descubre joyas ocultas de cientos de paises dentro de TikTok y graba tus momentos de viaje en tiempo real para tener la oportunidad de ganar Go Pro! 9 Terms and conditions:http://mrw.so/50Pgm4
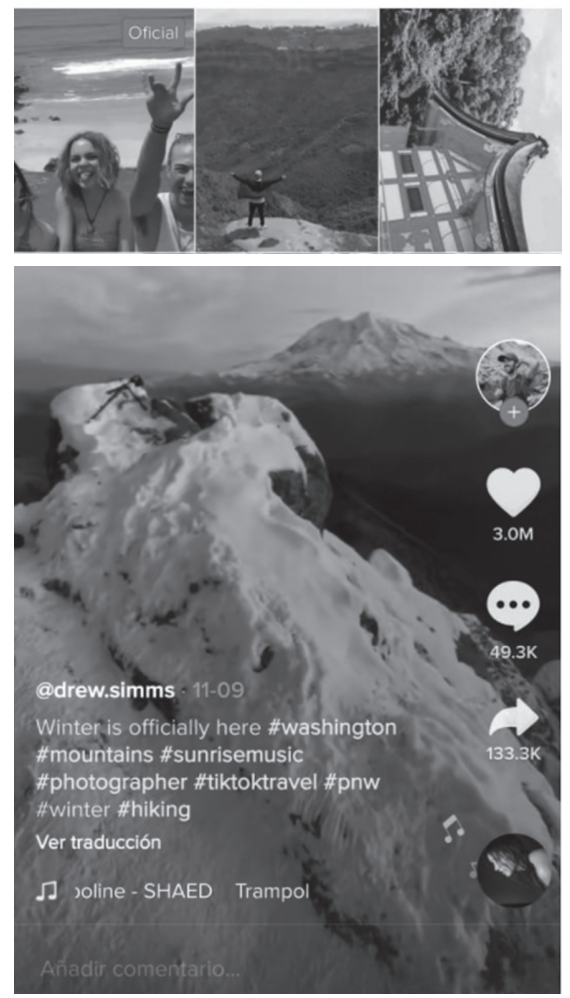

Fuente: Página oficial de Tik Tok, 3 de enero de 2021.

de la cuenta de @drew.simms. Las métricas responden de un total de 3 millones de 'me gusta', 49,3 mil comentarios y 133,3 mil shares. La grabación fue realizada en el Monte Washington, ubicado al norte de los Estados Unidos, en el Condado de Coös, New Hampshire.

\section{Conclusiones y discusión}

Se puede concluir que las plataformas sociales son una herramienta efectiva para la difusión de las experiencias de los viajeros, su alcance masivo y bajo costo agilita el proceso de intercambio de información entre los usuarios. Además, de la influencia que genera en la decisión de elegir un destino.

Existe un importante segmento de viajeros jóvenes que buscan viajes que les permita estar en contacto con la naturaleza y tienen como primer medio de búsqueda de información las redes sociales. Es aquí donde TikTok ha tomado especial relevancia, convirtiéndose en una de las plataformas más empleadas durante 2019 y 2020 por la generación Z y los millennials. Las vivencias compartidas a través de los vídeos cortos sintetizan los paisajes y las posibles experiencias que pueden vivir.

Emplear TikTok como una herramienta que promueve a los jóvenes a involucrarse con el entorno natural y volcarse a vivir experiencias holísticas genera un impacto relevante sobre la cultura de los viajes. Además, permite identificar a los espacios naturales como el camino para escapar del estrés, depresión y todo cuanto puede generar un espacio cerrado. En medio de un mundo digitalizado que gira alrededor de un móvil u ordenador, este es un efecto positivo que se debe rescatar en medio del caos que el mundo vive.

El turismo sostenible se ha convertido en una necesidad, en especial en tiempos donde la seguridad sanitaria sale del control gubernamental y privado. Las familias buscan espacios donde poder compartir tranquilos y sin presión, aislados de las aglomeraciones y posibles contagios. Es así que en el 2021 se puede seguir ratificando que esta forma de hacer turismo es una de las más seguras para todas las edades; sin dejar fuera el 
importante aporte que genera para el bienestar del ser humano.

TikTok representa una oportunidad para impactar positivamente en la forma de hacer turismo, es esencial darle un giro productivo y relevante al uso que normalmente se da a esta plataforma. Hay que emplearlo como un medio de difusión y aprovechando el alcance de audiencia y usuarios que posee. Actores de gobierno, empresarios y colectivo tienen a la mano una plataforma que facilita el fomento de uno de los pocos sectores que se encarga de recrear experiencias para el esparcimiento de las personas y además de reactivar uno de los sectores más golpeados en el mundo, producto de la pandemia.

La investigación no se encuentra exenta de limitaciones. Se considera oportuno realizar el análisis de las métricas de TikTok por país, debido a que la implementación de la plataforma se encuentra desarrollada en distintos niveles por parte de los usuarios y los establecimientos. No obstante, la red social por el momento cuenta con un único mecanismo de análisis de las estadísticas y es la analítica de las cuentas Pro, que al ser de carácter privado de los usuarios no es posible realizar un análisis profundo de las métricas.

\section{Bibliografía}

Alzate, J. y Orozco, J. (2018). Stakeholders, actores estratégicos en la construcción de marca. Anagramas - Rumbos y Sentidos de La Comunicación, 16(32), 95-109. DOI: https:// doi.org/10.22395/angr.v16n32a6

Cabrer, B., Szmulewicz, P., García, G., Gutiérrez, C. y Sancho, A. (2010) . El turismo sostenible, un reto para los destinos emergentes de la región de los ríos en el sur de Chile. Aportes y Transferencias, 14(2), 49-60. Disponible en: https://www.redalyc.org/pdf/276/27621284003.pdf

Carrión, R. (2020). El TikTok, de adicción juvenil a herramienta de aprendizaje. Investigando. DOI: 10.13140/ RG.2.2.29177.49764

Dai, L. \& Chen, P. (2019). Reasons for the popularity of Tik Tok, the shortages and the ways forward. Advances in Social Science, Education and Humanities Research, 345, 544 - 548. DOI: 10.2991/isemss-19.2019.105

Dwivedi, Y. K., Ismagilova, E., Hughes, D. L., Carlson, J., Filieri, R., Jacobson, J., Jain, V., Karjaluoto, H., Kefi, H., Krishen, A. S., Kumar, V., Rahman, M. M., Raman, R., Rauschnabel, P. A., Rowley, J., Salo, J., Tran, G. A. \& Wang, Y. (2020). Setting the Future of Digital and Social Media Marketing Research: Perspectives and Research Propositions. International Journal of Information Management, online, 1-37. DOI: http://dx.doi.org/10.1016/j.ijinfomgt.2020.102168
Feng, Y., Chen, C. \& Wu, S. (2019). Evaluation of Charm Factors of Short video User Experience using FAHP-A Case Study of TikTok APP. In IOP Conference Series: Materials Science and Engineering, 688(5), 55-68. DOI: 10.1088/1757899X/688/5/055068

Haenlein, M., Anadol, E., Farnsworth, T., Hugo, H., Hunichen, J. \& Welte, D. (2020). Navigating the New Era of Influencer Marketing: How to be Successful on Instagram, TikTok, \& Co. California Management Review, 63(1), 5-25. DOI: https://doi.org/10.1177/0008125620958166

Herrera, A. (2020). La comunicación como factor clave en la experiencia del consumidor para la fijación del precio. Revista Internacional de Investigación en Comunicación aDResearch ESIC, 21(21), 84-97. DOI: https://doi.org/10.7263/ adresic-021-05

Li, Y., Xu, X., Song, B. \& He, H. (2020). Impact of Short Food videos on the Tourist Destination Image-Take Chengdu as an Example. Sustainability, 12(17). DOI: https://doi.org/10.3390/su12176739

Linares, H. y Morales Garrido, G. (2014). Del desarrollo turístico sostenible al desarrollo local. Su comportamiento complejo. PASOS. Revista de Turismo y Patrimonio Cultural, 12(2), 453-466. Disponible en: https://www.redalyc.org/ articulo.oa?id=88130205003 
Liu, F, Zhu, Z., Chen, H. \& Li, X. (2020). Beauty in the eyes of its beholders: Effects of design novelty on consumer preference. Journal of Retailing and Consumer Services, 53. DOI: https://doi.org/10.1016/j.jretconser.2019.101969

Omar, B. \& Dequan, W. (2020). Watch, Share or Create: The Influence of Personality Traits and User Motivation on TikTok Mobile video Usage. International Association of Online Engineering, 14(4). DOI: https://doi.org/10.3991/ijim. v14i04. 12429

Pine, B. J. \& Gilmore, J. H. (1998). Welcome to the experience economy. Harvard Business Review, 97-105.

Rey, J. P. (2020). TikTok mucho más que bailecitos y centennials. Interactiva: Revista de la comunicación y el marketing digital, (190), 56-63.

Rialti, R., Caliandro, A., Zollo, L. y Ciappei, C. (2018). Comunidades de marca de medios sociales y co-creación de experiencias. Spanish Journal of Marketing - ESIC, 22(2), 122 141. DOI: https://doi.org/10.1108/SJME-03-2018-0011

Shen, X. (2019). Analysis on the Innovation of Rural Tourism Marketing Strategy-Taking the TikTok as an Example. Ad- vances in Economics, Business and Management Research, 109, 551-554. DOI: 10.2991/aebmr.k.191217.099

Shutsko, A. (2020). User-Generated Short video Content in Social Media. A Case Study of TikTok. Meiselwitz G. (eds) Social Computing and Social Media. Participation, User Experience, Consumer Experience, and Applications of Social Computing. HCII 2020. Lecture Notes in Computer Science, 12195, 108-125. Springer, Cham. DOI: https://doi. org/10.1007/978-3-030-49576-3_8

Ying, K. \& Krishnapillai, G. (2018). Young Domestic Tourists-Are They Motivated to Travel?. Advanced Science Letters, 24(5), 3444-3449. DOI: 10.1166/asl.2018.11405

Zhang, H., Gordon, S., Buhalis, D. \& Ding, X. (2018). Experience Value Co creation on Destination Online Platforms. Journal of Travel Research, 57(8), 1093-1107. DOI: https://doi.org/10.1177/0047287517733557

Zhang, Z. (2020). Infrastructuralization of TikTok: transformation, power relationships, and platformization of video entertainment in China. Media, Culture E Society, online. DOI: $10.1177 / 0163443720939452$

\section{Referencias web}

Hotelmize (2019). Las 5 mejores redes sociales para agencias de viajes y cómo sacarles partido. Recuperado de: https://www.hotelmize.com/es/blog/las-5-mejores-redessociales-para-agencias-de-viajes-y-como-sacarles-partido/ Organización Mundial del Turismo (2020). La OMT pone en marcha un programa de asistencia técnica para la recu- peración del turismo. Recuperado de: www.unwto.org/es/ news/la-omt-pone-en-marcha-un-programa-de-asistenciatecnica-para-la-recuperacion-del-turismo

TikTok (2020). Estadísticas. Recuperado de: https://newsroom.tiktok.com/en-us/pro-accounts-offer-new-insightsto-avid-creators/ 